\title{
Corrigendum: Gene-Related Cerebellar Neurodegeneration in SCA3/MJD: A Case-Controlled Imaging-Genetic Study
}

\author{
Huirong Peng ${ }^{1}$, Xiaochun Liang ${ }^{1}$, Zhe Long ${ }^{2}$, Zhao Chen ${ }^{1}$, Yuting Shi ${ }^{1}$, Kun Xia ${ }^{3}$, \\ Li Meng ${ }^{4}$, Beisha Tang 1,3,5,6,7,8,9, Rong Qiu ${ }^{10 *}$ and Hong Jiang ${ }^{1,3,5,6,11 *}$
}

${ }^{1}$ Department of Neurology, Xiangya Hospital, Central South University, Changsha, China, ${ }^{2}$ Department of Neurology, Second Xiangya Hospital, Central South University, Changsha, China, ${ }^{3}$ Laboratory of Medical Genetics, Central South University, Changsha, China, ${ }^{4}$ Department of Radiology, Xiangya Hospital, Central South University, Changsha, China, ${ }^{5}$ National Clinical Research Center for Geriatric Diseases, Central South University, Changsha, China, ${ }^{6}$ Key Laboratory of Hunan Province in Neurodegenerative Disorders, Central South University, Changsha, China, ${ }^{7}$ Parkinson's Disease Center, Beijing Institute for Brain Disorders, Beijing, China, ${ }^{8}$ Collaborative Innovation Center for Brain Science, Shanghai, China, ${ }^{9}$ Collaborative Innovation Center for Genetics and Development, Shanghai, China, ${ }^{10}$ School of Information Science and Engineering, Central South University, Changsha, China, ${ }^{11}$ Department of Neurology, Xinjiang Medical University, Urumchi, China

Keywords: spinocerebellar ataxia 3 , gray matter, white matter, ${ }^{1} \mathrm{HMRS}$, imaging genetics study

\section{A Corrigendum on}

Gene-Related Cerebellar Neurodegeneration in SCA3/MJD: A Case-Controlled Imaging-Genetic Study

OPEN ACCESS

Approved by:

Frontiers Editorial Office,

Frontiers Media SA, Switzerland

*Correspondence:

Rong Qiu

qiurongrong@126.com

Hong Jiang

jianghong73868@126.com

Specialty section:

This article was submitted to

Neurodegeneration,

a section of the journal

Frontiers in Neurology

Received: 07 January 2020 Accepted: 09 January 2020

Published: 07 February 2020

Citation:

Peng H, Liang $X$, Long Z, Chen Z, Shi Y, Xia K, Meng L, Tang B, Qiu R and Jiang $H$ (2020) Corrigendum:

Gene-Related Cerebellar

Neurodegeneration in SCA3/MJD: A Case-Controlled Imaging-Genetic

Study. Front. Neurol. 11:30. doi: 10.3389/fneur.2020.00030 by Peng, H., Liang, X., Long, Z., Chen, Z., Shi, Y., Xia, K., et al. (2019). Front. Neurol. 10:1025. doi: 10.3389/fneur.2019.01025

There is an error in the Funding statement. The correct number for the National Natural Science Foundation of China is No. 81771231 to HJ and No. 81600995 to YS.

Additionally, we neglected to include the funder the National Natural Science Foundation of China, No. 81974176 to HJ; No. 81901169 to ZC. The corrected funding statement appears below.

\section{FUNDING}

We declared that there were no financial interests in this study. This study was supported by the National Key Research and Development Program of China (Nos. 2016YFC0901504 and 2016YFC0905100 to HJ; No. 2016YFC1306000 to BT), the National Natural Science Foundation of China (Nos. 81771231 and 81974176 to HJ; No. 81600995 to YS; No. 81901169 to ZC), the National Natural Science Foundation of Hunan Province (No. 2019JJ40363 to RQ), Key Research and Development Program of Hunan Province (No. 2018SK2092 to HJ), Scientific Research Foundation of Health Commission of Hunan Province (No. B2019183 to HJ), The Clinical and rehabilitation fund of Peking University Weiming Biotech Group (No. xywm2015I10 to HJ).

The authors apologize for this error and state that this does not change the scientific conclusions of the article in any way. The original article has been updated.

Copyright (๑ 2020 Peng, Liang, Long, Chen, Shi, Xia, Meng, Tang, Qiu and Jiang. This is an open-access article distributed under the terms of the Creative Commons Attribution License (CC BY). The use, distribution or reproduction in other forums is permitted, provided the original author(s) and the copyright owner(s) are credited and that the original publication in this journal is cited, in accordance with accepted academic practice. No use, distribution or reproduction is permitted which does not comply with these terms. 